\title{
CME Oncology in the emergency room SAQs (91576): Self-assessment questionnaire
}

\author{
Author: Susan O'Reilly
}

SAQs and answers are ONLINE for RCP fellows and collegiate members

The SAQs printed in the CME section can only be answered online to achieve external CPD credits. Any comments should be sent in via email only: clinicalmedicine@rcplondon.ac.uk

\section{Format}

SAQs follow a best of five format in line with the MRCP(UK) Part 1 exam. Candidates are asked to choose the best answer from five possible answers.

\section{The answering process}

1 Go to www.rcplondon.ac.uk/SAQ

2 Log on using your usual RCP username and password

3 Select the relevant $\mathrm{CME}$ question paper

4 Answer all 10 questions by selecting the best answer from the options provided

5 Once you have answered all the questions, click on Submit

\section{Registering your external CPD credits}

Carrying out this activity allows you to claim two external CPD credits. These will be automatically transferred to your CPD diary, where you can review the activity and claim your points.

1 A 57-year-old woman presented to the emergency department having woken up feeling generally unwell. Her temperature, taken at home, was $38^{\circ} \mathrm{C}$. She had no other symptoms. 9 days previously she received a course of adjuvant chemotherapy, following a diagnosis of $\mathrm{T}_{2} \mathrm{~N}_{1} \mathrm{M}_{0}$ breast carcinoma earlier in the year. Physical examination revealed a temperature of $37.6^{\circ} \mathrm{C}$ but was otherwise unremarkable.

\section{What is the most appropriate management?}

(a) Take blood for urgent full blood count (FBC) and, if this reveals a neutrophil count of $<0.5 \times 10^{9} /$, take blood cultures and start immediate broad spectrum antibiotics.

(b) Take blood for FBC, biochemistry, C-reactive protein (CRP), lactate dehydrogenase (LDH) and blood cultures. Monitor vital signs closely and if temperature rises to $>38^{\circ} \mathrm{C}$, start broad spectrum antibiotics. (c) Take blood for FBC, biochemistry, CRP, LDH and blood cultures. Send patient for chest X-ray (CXR) and perform urinalysis. If there is no obvious source of infection, start broad spectrum antibiotics.

(d) Take blood for FBC, biochemistry, CRP, LDH and blood cultures, and start immediate broad spectrum antibiotics.

(e) Take blood for FBC, biochemistry, CRP, LDH and blood cultures. Admit patient and await result of blood cultures to guide choice of antibiotic therapy.

2 With regards to febrile neutropenia in a patient following cytotoxic chemotherapy, which statement is true?

(a) In patients for whom a bacterial source is identified, Gram negative pathogens are the most common causative organisms.

(b) Primary prophylaxis with quinolones reduces the incidence of neutropenic sepsis and short term mortality.

(c) Primary prophylaxis with granulocyte-colony stimulating factor (GCSF) is recommended for patients receiving chemotherapy regimens with a risk of febrile neutropenia of $>10 \%$.

(d) Deaths from neutropenia sepsis in the UK have been falling in the past decade.

(e) In patients with febrile neutropenia, blood cultures usually identify the causative pathogen.

3 A 75-year-old man presented to the emergency department with a swollen right leg, following recent commencement of palliative chemotherapy for multiple liver metastases from a colorectal carcinoma. Doppler ultrasound confirmed deep vein thrombosis.

Blood results: haemoglobin $(\mathrm{Hb})$

white blood cells (WBC)

platelets

estimated glomerular filtration rate (eGFR)

$9.9 \mathrm{~g} / \mathrm{dl}$

$3.2 \times 10^{9} /$

$80 \times 10^{9} / 1$

$40 \mathrm{ml} / \mathrm{min}$
Which of the following is the most appropriate management?

(a) Start low-molecular-weight heparin and convert, after a few days, to oral anticoagulant.

(b) Anticoagulation is not indicated because of poor prognosis

(c) Defer anticoagulation until platelet count is $>150 \times 10^{9} /$.

(d) Start unfractionated heparin in view of poor renal function.

(e) Treat with low molecular weight heparin for a minimum of 6 months. 
4 With regards to venous thromboembolism (VTE) in malignancy, which statement is true?

(a) VTE is most common in patients with cancers of the pancreas, bowel and breast.

(b) Ambulant patients with pancreatic cancer should receive pharmacological VTE prophylaxis.

(c) Patients found to have an incidental, asymptomatic pulmonary embolus on staging computed tomography (CT) scans should receive anticoagulation therapy.

(d) Recurrent VTE, despite anticoagulation, occurs in around $17 \%$ of patients on low-molecular-weight heparin.

(e) VTE is a serious complication in cancer patients and all patients should be admitted to hospital for treatment.

5 A 65-year-old man presented to the emergency department with recent onset severe low back pain and a 'heavy' sensation in his legs. One year previously he had had a right lower lobectomy for squamous carcinoma of lung, but he was not known to have any metastatic disease. Physical examination revealed slightly reduced power in both legs (grade 4/5), but no sensory loss.

\section{What should be the next step?}

(a) X-ray lumbar spine.

(b) Lie patient flat, start high dose steroids and arrange immediate magnetic resonance imaging (MRI) lumbar spine.

(c) Arrange urgent MRI of the whole spine (within 1 week).

(d) Lie patient flat, start high dose steroids and arrange a whole spine MRI within 24 hours.

(e) Arrange urgent bone scan to assess whether patient has bone metastases.

6 A 45-year-old woman on palliative endocrine therapy for metastatic breast cancer (lung and bone) was admitted to the acute medical unit with recent onset severe pain in mid-dorsal spine. She had no other symptoms and physical examination was normal. She had been assessed recently in the oncology clinic where scans showed that her metastatic disease seemed stable on treatment. Magnetic resonance imaging (MRI) revealed spinal cord compression at T8. Bed rest and high dose steroids were started.

\section{What is the most appropriate further management?}

(a) Offer single fraction (8 Gy) radiotherapy to dorsal spine.

(b) Arrange urgent decompressive surgery.

(c) Offer long-course radiotherapy (20 Gy in 10 fractions).

(d) Contact Metastatic Spinal Cord Compression network coordinator to discuss suitability for decompressive surgery, followed by radiotherapy.

(e) Offer symptomatic treatment only in view of extraosseous metastases.

7 A 68-year-old man presented to the acute medical unit with mild, intermittent, 'crampy' abdominal pains and five loose stools per day. He had no other symptoms. 10 days previously he had received 5 -fluorouracil chemotherapy. Physical examination was normal, with a heart rate of 75 beats per minute and blood pressure of $120 / 75 \mathrm{mmHg}$.
Investigations:

haemoglobin $(\mathrm{Hb})$

$9.9 \mathrm{~g} / \mathrm{dl}$

white blood cell count (WBC)

$3.4 \times 10^{9} / 1$

neutrophils

$1.1 \times 10^{9} / 1$

platelets

biochemistry

$112 \times 10^{9} / 1$

normal

\section{What is the most appropriate management?}

(a) Start on loperamide as an outpatient and advise oncologist of symptoms so testing for dihydropyrimidine dehydrogenase (DPD) deficiency can be arranged.

(b) Admit for intravenous fluids.

(c) Admit to hospital and start octreotide.

(d) Start loperamide and advise patient that if symptoms worsen, or do not settle within 48 hours, he should return for further assessment and possible admission.

(e) Admit to hospital for observation and start oral loperamide.

8 Regarding diarrhoea in cancer patients, which of the following statements is true?

(a) Clostridium difficile should only be suspected as a cause if the patient has received prior antibiotic therapy.

(b) Diarrhoea is less common after targeted biological therapies than after chemotherapy.

(c) Diarrhoea is most commonly associated with cytotoxic chemotherapy drugs used to treat cancers of the gastrointestianal tract.

(d) Assays to detect enzyme deficiency status should be performed prior to treatment with treatments likely to cause diarrhoea.

(e) UGT1A1 polymorphism is associated with an increased risk of diarrhoea after 5 -fluorouracil chemotherapy.

9 A 42-year-old woman with metastatic breast cancer was admitted to the acute medical unit with a history of increasingly severe headaches. She was fully active and recent computed tomography (CT) scans showed that her lung metastases were responding to palliative endocrine therapy. A brain CT scan revealed a solitary right frontal mass with surrounding oedema.

\section{What is the most appropriate next step?}

(a) Immediate transfer to neurosurgical centre.

(b) Start high dose steroids and arrange urgent magnetic resonance imaging (MRI) brain.

(c) Lumbar puncture to rule out meningeal metastases in addition to brain metastasis.

(d) Start high dose steroids and anticonvulsants and arrange urgent MRI brain.

(e) Urgent referral to oncologists for whole brain radiotherapy.

10 In patients with brain metastases, which of the following statements is true?

(a) Whole brain radiotherapy has been shown in randomised clinical trials to prolong survival for patients with multiple brain metastases. 
(b) After resection of a solitary brain metastasis, whole brain radiotherapy reduces local recurrence rates.

(c) Surgical resection is superior to stereotactic radiosurgery (SRS) for patients with a solitary brain metastasis.

(d) There is no role for chemotherapy in the treatment of brain metastases.

(e) Brain metastases occur in up to $60 \%$ of cancer patients.

\section{CME Genetics SAQs}

Answers to the CME SAQs published in Clinical Medicine August 2014

$\begin{array}{llllllllll}\text { Q1 } & \text { Q2 } & \text { Q3 } & \text { Q4 } & \text { Q5 } & \text { Q6 } & \text { Q7 } & \text { Q8 } & \text { Q9 } & \text { Q10 }\end{array}$

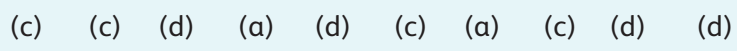

\section{Erratum}

\section{Delirium: a synthesis of current knowledge}

\section{Barbara C van Munster and SE de Rooji}

Clinical Medicine 2013;14:192-5.

An error was introduced to Table 1 of this article. A corrected table is printed below.

Table 1. Summarised criteria for delirium from recent versions of the Diagnostic and Statistical Manual of Mental Disorders., ${ }^{1,2}$

\section{DSM IV-R criteria}
A Disturbance of consciousness with reduced attention
B Change in cognition or a new perceptual disturbance
C Acute development (usually hours to days) and tends to fluctuate over 1 day

D Caused by a somatic factor, medication intoxication or withdrawal

\section{DSM-5 criteria}

A Disturbance in attention

C Change in an additional cognitive domain, not accounted for by another neurocognitive disorder

B Acute development and fluctuation over a day and not solely the result of another neurocognitive disorder

D Disturbances in A and C must not be occurring in the context of a severely reduced level of arousal, such as coma

E Caused by a somatic factor, medication intoxication or withdrawal

DSM = Diagnostic and Statistical Manual of Mental Disorders. Changes in DSM-5 from DSM-IV shown in italics.

\section{References}

1 American Psychiatric Association. Diagnostic and Statistical Manual of Mental Disorders: DSM IV-TR. Washington, DC: American Psychiatric Publishing, 2000.

2 American Psychiatric Association. Diagnostic and Statistical Manual of Mental Disorders: DSM-5. Arlington, VA: American Psychiatric Publishing, 2013. 